www.jmscr.igmpublication.org

Impact Factor (SJIF): 6.379

Index Copernicus Value: 79.54

ISSN (e)-2347-176x ISSN (p) 2455-0450

crossrefDOI: https://dx.doi.org/10.18535/jmscr/v6i8.126

Journal Of Medical Science And Clinical Research

\title{
Coronary Angiographic (CAG) Findings between Diabetic and non diabetic Patients in Coronary artery disease: A Comparative Study
}

\author{
Authors
Dr Rajiv Girdhar ${ }^{1}$, Dr Yogesh Kothari ${ }^{2}$, Dr Anuj Sanjay Kamat ${ }^{3}$, Dr Ram Anil Raj ${ }^{4}$, Dr Bryan Jacob Koithara \\ ${ }^{1}$ DM Cardiology PG, ${ }^{2} \mathrm{HOD}$ and Professor in Cardiology \\ ${ }^{3}$ MD Medicine PG Student, ${ }^{4}$ Professor in Cardiology, ${ }^{5}$ Internship in Cardiology \\ Rajarajeswari Medical College and Hospital, Mysore Road, Bangalore 560074, India
}

\begin{abstract}
Introduction: Coronary artery disease (CAD) accounts for the major chunk of mortality in diabetes. Coronary angiography or arteriography remains the "gold-standard" technique for diagnosing and evaluating CAD.

Material and Methods: The present study was undertaken at Rajarajeswari Medical College and Hospital, Bangalore, India between the periods of $1^{\text {ST }}$ December- 2016 to $31^{\text {st }}$ June - 2018.

Percutaneous coronary angiography (CAG) was Performed in 200 consecutive patients with suggestive of ischaemic chest pain. The population consisted of of which 75 cases are diabetic and 125 cases are nondiabetic
\end{abstract}

Results: We observed positive angiographic lesion among both groups comparing site \& number of vessel(s) involvement also average percentage of stenosis. The presence of coronary risk factors was not significantly different between the two populations. Total positive angiographic lesion was 131 (65.5\%) in both groups. Among the Diabetes mellitus patients positive CAG finding 61 (81.33\%). The recognized lesions were single vessel disease (SVD) 16 (26.24),double vessel disease (DVD) 25 (40.98\%), triple vessel disease (TVD) 20 (32.78\%), diffuse lesions $6(9.8 \%)$ and average vessel stenosis $82.63 \%$. On the other hand, total positive angiographic lesion was $70(56 \%)$ in non--diabetic group; among them single vessel disease (SVD) 23 (32.85\%), double vessel disease (DVD) 28(40\%), triple vessel disease (TVD) 19 (27.15\%), no diffuse lesions was found and average vessel stenosis was $78.03 \%$. The results of the angiographic finding suggest that diabetic patients have a higher incidence of coronary heart disease (CHD), DVD, TVD, diffuse lesion \& marked stenosis of coronary vessel than nondiabetic patient.

Conclusion: $C A D$ in diabetics had considerably higher percent of severe and unpredictable presentation. This increased frequency of complex lesion morphology is more difficult to treat by definitive intervention like percutaneous transluminal coronary angioplasty (PCI) \& coronary artery bypass graft (CABG). Diabetics have a higher risk factor profile and poor clinical outcome. Early diagnosis and appropriate management will reduce the risk of complication after the onset of disease.

Keywords: Coronary artery disease, Coronary angiogram, Diabetes mellitus, Non-diabetics.

\section{Introduction}

Coronary artery disease (CAD) accounts for the major chunk of mortality in diabetes. Diabetes mellitus (DM) is a well-established risk factor for development of coronary artery disease (CAD) ${ }^{1,2}$. Coronary atherosclerosis is not only more 
prevalent in diabetic patients but also more severe. The reported prevalence of coronary artery disease in diabetic patients ranges from $9.5 \%$ to $55 \%{ }^{3,4}$ Whereas prevalence of $1.6 \%$ to $4.1 \%$ have been observed in the general population. 5,6

Incidence of heart diseases \& ischaemic heart mortality was shown to be 4 times higher in people with Type-2 DM. ${ }^{7}$ Type -1 DM was seen to be associated with at least a 10 fold increase as compared with people without diabetes ${ }^{8}$ In people with DM 40\%, 15\%, 10\% death occur due to ischaemic heart disease (IHD), other heart diseases \& cerebrovascular disease (CVD) respectively. ${ }^{9}$ Several in vivo and postmortem studies have shown that diabetic patients have more diffuse and severe coronary artery disease than the general population. ${ }^{1,10,11}$

In addition, the relative risk of myocardial infarction (MI) is greater in diabetic patients than in the normalpopulation. ${ }^{12}$ The cause of this difference in the diabetic population is not well understood. But it is suggested that diabetic patients have several hematologic, and metabolic abnormalities not present in their non diabetic counterparts $^{13,14,15}$ that may predispose them to formation of more complex plaque. To date, very few studies, have attempted to explain these differences between diabetic and non diabetic patients in our country. Thus this prospective cross sectional study was designed to find out the morphological pattern of coronary lesion among diabetes mellitus and to compare with non diabetic cases. Hence the study was undertaken.

\section{Materials and Methods}

200 cases of males and females, who presented in the department of Cardiology, Rajarajeshwari Medical college Hospital (RRMCH), Bangalore, from $1^{\text {ST }}$ December- 2016 to $31^{\text {st }}$ June -2018. were included in this study. Involved patients were selected on the basis of inclusion and exclusion criteria as mentioned below.

\section{Study population}

Inclusion criteria: All patients clinically diagnosed or documented to have CAD, who required coronary angiography (CAG) was taken as study population. Informed consent was taken from all patients.

\section{The grouping of study population}

The study population was divided into two groups as follows

Group-1: Patients presented with features of ischaemic heart disease (IHD) \& having DM (DM group)

Group-2: Patients presented with features of ischaemic heart disease (IHD) but without DM (non DM group)

\section{Criteria's for diagnosis of DM}

Group-1 (Diabetic CAD) Previously known diabetic or first time detected diabetic by American diabetic association (ADA) criteria, 2007, presenting with CAD. ${ }^{16}$

Criteria's for diagnosis of non DM cases Patient do not meet the above ADA criteria's for confirm the diagnosis of DM.

Criteria's for coronary artery disease (CAD) \&Coronary Angiography (CAG)

1) Chronic stable angina pectoris with positive E.T.T (with or without previous MI)

2) Unstable angina pectoris

3) Atypical chest pain with positive E.T.T

4) After acute MI (with or without persistent angina)

5) Asymptomatic patient with noninvasive evidence of myocardial ischaemia (ECG, ECHO)

\section{Exclusion Criteria}

1) Patient with hypertrophic or dilated cardiomyopathy

2) Patient with valvular heart disease

3) Patient with congenital heart disease

4) Patients having impaired fasting glucose level presenting with CAD. (FPG $<126 \mathrm{mg} / \mathrm{dl}$ But>110 mg/dl, PP-PG 140$200 \mathrm{mg} / \mathrm{dl}$ ) 
On recruiting the subjects into Group 1 and Group 2 following protocol is followed. History, Clinical Examination, Pt. Stabilization, Anthropometric Measurement, Routine investigations, Specific investigations including Echocardiography Procedures, definitions and criteria were used in the study as per standard protocol (JNC-7, American diabetic association (ADA) criteria and others). ${ }^{17,18}$

\section{Coronary Angiographic (CAG) Procedure}

A comprehensive analysis of Coronary Angiogram (CAG) was done; severity \& extent of arterial disease were measured by eye estimation. The pre requisites for $\mathrm{CAG}$ were followed according to the hospital protocol, then morphological characteristics of lesion was analyzed
a) Positive CAG- taken when coronary artery stenosis $50 \%$
b) Negative CAG- taken when coronary artery stenosis $<50 \%$

According to branches of coronary artery involvement

1) Single vessel disease (SVD) -one coronary artery involve

2) Double vessel disease (DVD)- two coronary artery involve

3) Triple vessel disease (TVD) -three coronary artery involve

4) Diffuse lesion -diffusely involved one or more coronary artery

\section{Statistical analysis}

After processing of all available information, statistical analysis of their significance was done. The patients were grouped into those with \& without DM having CAG. All parametric values were expressed as mean \& nonparametric values were expressed in percentage (\%).The significance of difference between two groups were determined by using unpaired student's ' $t$ ' test, Pearson's chi-square test \& ' $z$ ' test where applicable. ' $\mathrm{P}$ ' value of less than 0.05 was considered to be significant.

\section{Results}

Total Number of patients studied -200

Diagnostic Yield (sensitivity) of CAG

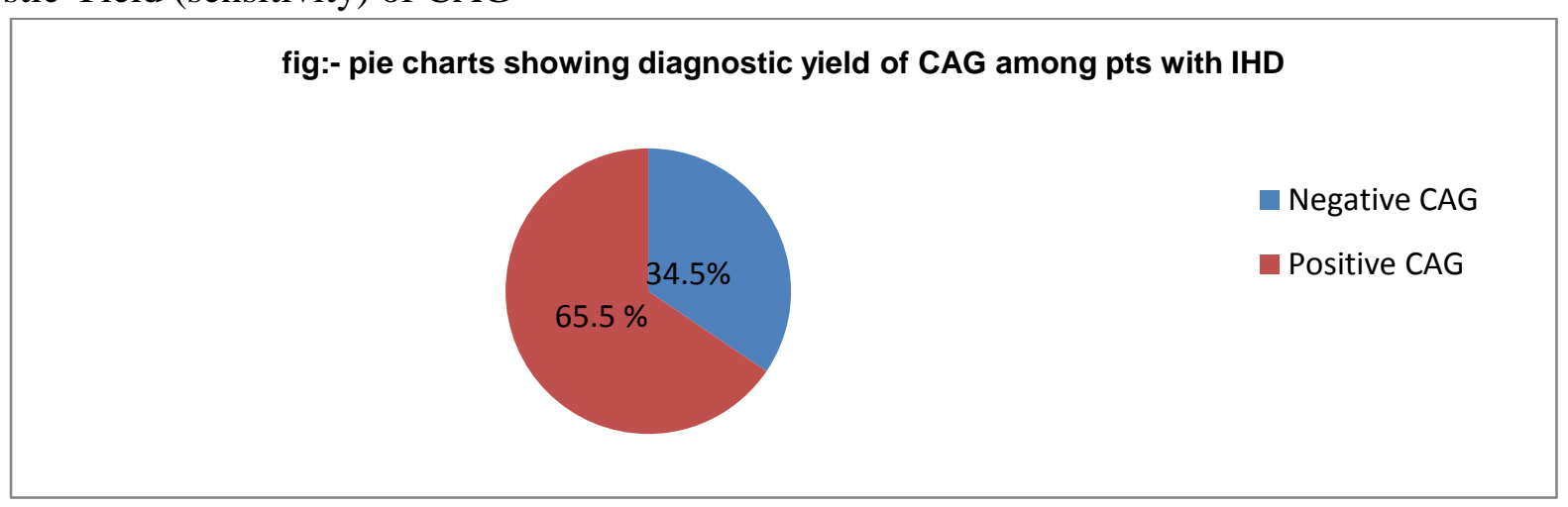

Distribution of patients

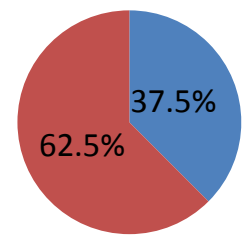

Fig-2: Pie chart showing distribution of patient undergone CAG 
Positive angiographic lesion among DM \& Non DM group

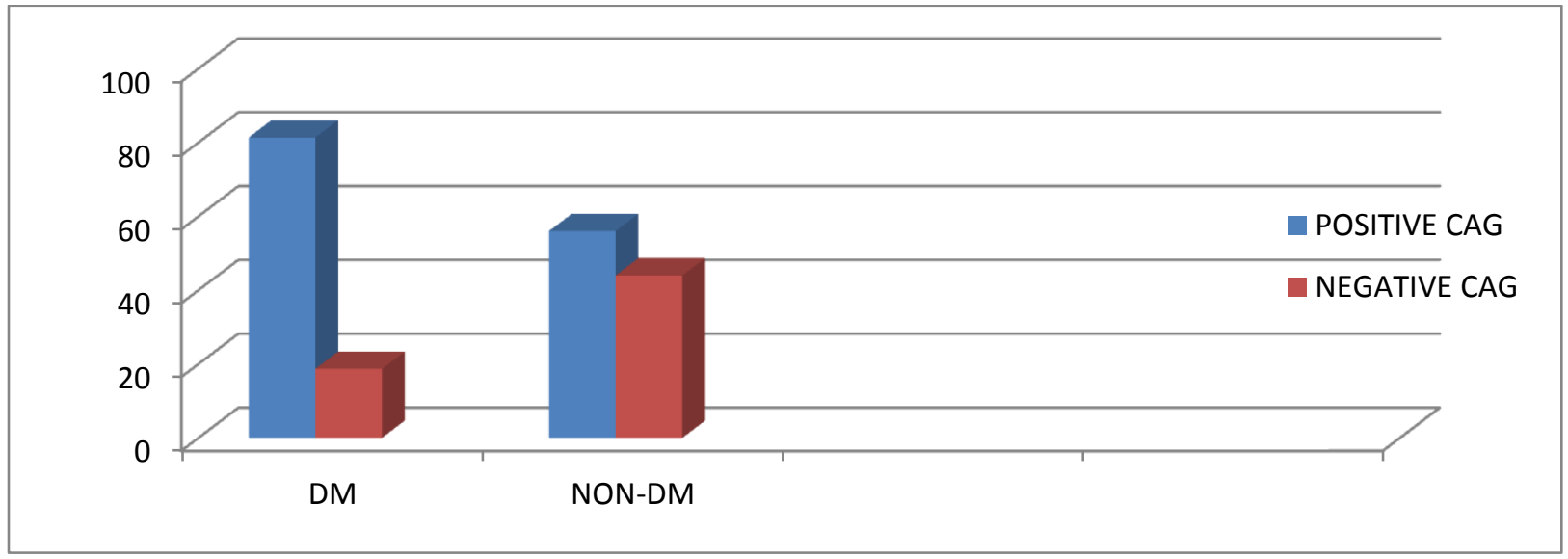

Fig-3: Bar diagram showing positive angiographic lesion

Pattern of Vessels involvement in DM and Non DM Patients. $(\mathrm{N}=200=100 \%)$

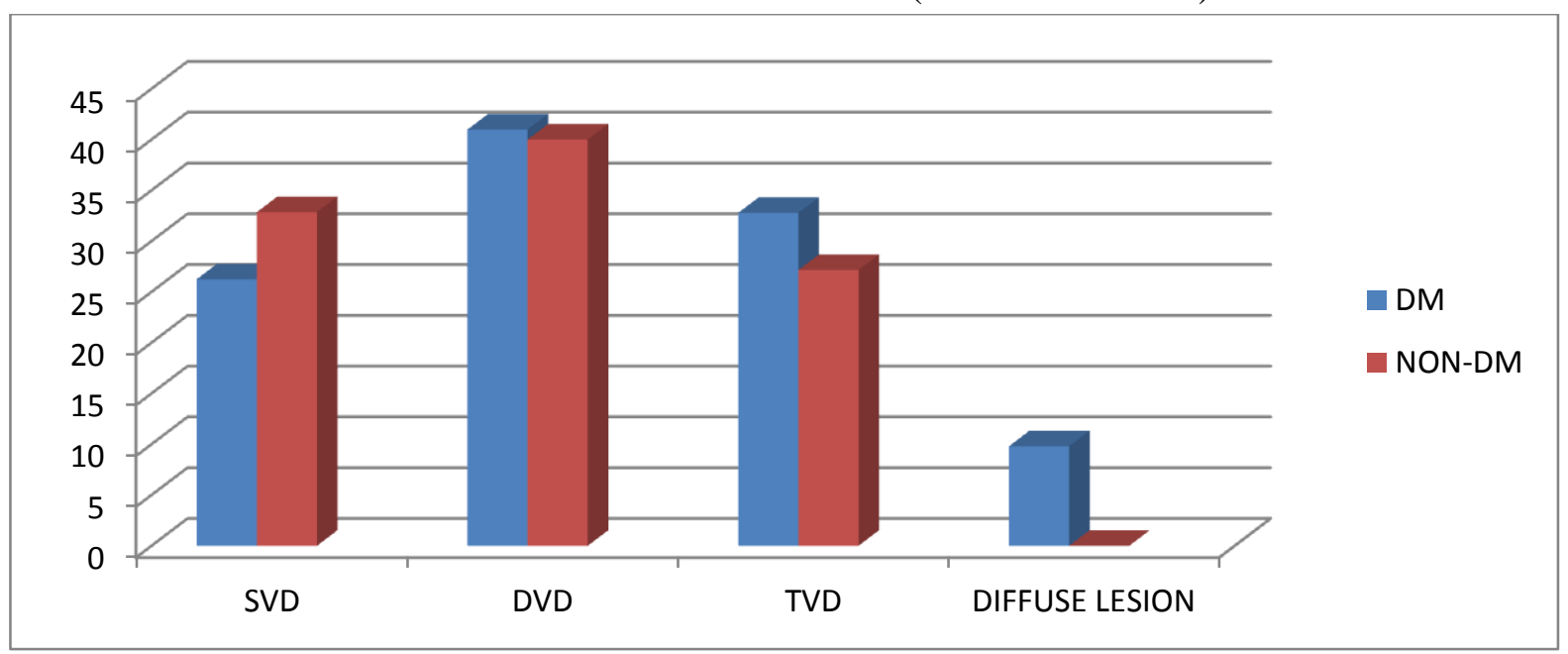

Fig-4: Bar diagram showing pattern of vessels involvement .Percentage of Vessel stenosis in DM and Non DM group

Table-1: shows percentage of vessel stenosis in DM and Non DM group

\begin{tabular}{|l|c|c|}
\hline & DM & NON-DM \\
\hline LMCA & $80 \%$ & $70 \%$ \\
\hline LAD & $81.48 \%$ & $79.41 \%$ \\
\hline LCX & $87.4 \%$ & $79.55 \%$ \\
\hline RCA & $84.65 \%$ & $83.15 \%$ \\
\hline Average & $82.63 \%$ & $78.03 \%$ \\
\hline
\end{tabular}

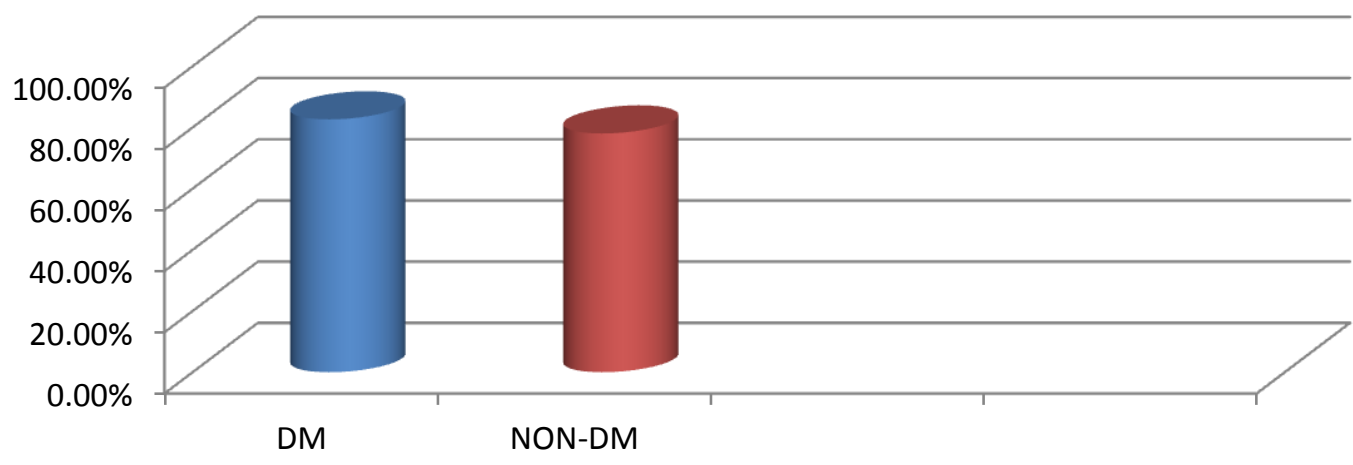

Fig-5: Bar diagram shows average vessel stenosis in DM and Non DM group 
Duration of diabetes

\section{Duration of diabetes}

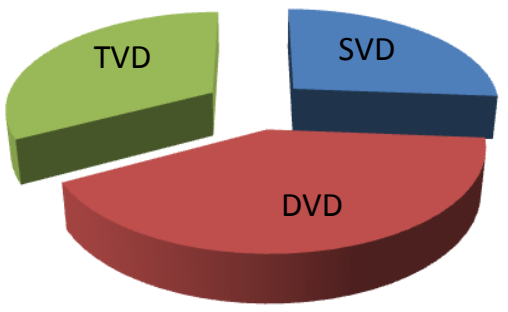

NEWLY DETECTED T2DM

- 5-10 YRS

MORE THAN 10 YRS

Fig-6: Pie chart showing duration of diabetes

\section{Discussion}

Cardiovascular diseases including CAD are more common among diabetics than among nondiabetics. In present study, there was no significance difference between age in two groups (Diabetic and non-diabetic) $\mathrm{P}>0.05$. Age difference between two groups and within groups among sex wise and list shows insignificance $(\mathrm{P}>0.05)$. Singer DE observed that, diabetic were older and noted that females are commonly involved, which agrees with our study. There was no much difference, in the involvement of younger age group ( $<40$ years) $(1.67 \%, 2 \%$, PNS). When age wise cut-off is seen, maximum events occurred in 50-59 years in both diabetics and non diabetics. Hence, age is an important risk factor for CHD in non-diabetic and diabetic population alike. $^{18}$

In the GUSTO-1 trial, when diabetics were considered, it was seen that, diabetic MI patients were older compared to non-diabetic MI's. But such results were not obtained in our study. GUSTO-1 trial says that females are commonly involved in diabetic group, which was Consistent with our results. ${ }^{19}$ This study demonstrates incidence \& difference of coronary heart disease (CHD), DVD, TVD, diffuse lesion among symptomatic diabetic and nondiabetic patient. The prevalence of coronary artery disease (more than $50 \%$ diameter stenosis) is more in DM patients $(81.33 \%)$ compared to their non DM counterparts $(56 \%)$. Moreno et $\mathrm{al}^{20}$ found the incidence of thrombus was higher in patients with diabetes than in patients without diabetes (62\% versus $40 \%$ ). Our study also demonstrates that diabetic patients had a higher prevalence of three-vessel disease (TVD) $(32.78 \%$ versus $26.19 \%)$ and lower prevalence of single vessel disease (SVD) $(26.24 \%$ versus $33.33 \%)$. Jose A Silva et $\mathrm{al}^{21}$ found diabetic patients had a higher prevalence of three-vessel disease (47\% versus $31 \%$ ) and lower prevalence of single-vessel disease $(18 \%$ versus $32 \%$ ) than non diabetic patients, although these differences were not statistically significant. In one large autopsy study, Waller et al ${ }^{11}$ reported that $91 \%$ of patients with adult-onset diabetes (type II) had severe (>75\%)narrowing of at least one major coronary artery and $81 \%$ had severe two- or three-vessel involvement .Our study demonstrates average vessel stenosis $82.63 \%$ in DM group as against $78.03 \%$ in the non diabetic in dividuals. Whether or not coronary atherosclerosis is more diffuse in diabetic patients is controversial. $^{11}$

The incidence of triple/MVD was significantly higher with the duration of diabetes $>10$ years. These findings correlate with the other study by Fox et al., ${ }^{28}$ showing the risk of coronary heart disease was 1.38 times higher for each 10 years increase in the duration of diabetes $(95 \%$ confidence interval, 0.99-1.92). Our study, coronary angiography revealed that the incidence of MVD in diabetics was much higher compared to non-diabetics which were only $16 \%$. This 
finding correlates with the other study conducted at CMC Vellore (1996) also showed that MVD was more common in diabetics $(87.5 \%$ vs. $79.6 \%)$ in 2 separate groups of 516 diabetic and nondiabetic patients. In our study $10.80 \%$ DM patients showed diffuse stenosis which was absent in the non DM group. In a study by Henry et al., ${ }^{22}$ and Sousa et al., ${ }^{23}$ there was increased the incidence of triple vessel disease, and more diffuse lesions were noted This finding was similar in other studies such as Mossavi et al., ${ }^{24}$ Uddin et al. ${ }^{25}$ Nicholls et al., ${ }^{26}$ and Rana et al., ${ }^{27}$ where they found the angiographic extent and severity of CAD was high in diabetic patients.

\section{Conclusion}

CAD in diabetics had considerably higher percent of severe and unpredictable presentation. This increased frequency of complex lesion morphology is more difficult to treat by definitive Intervention like percutaneous transluminal coronary angioplasty (PCI) \& coronary artery bypass graft (CABG). Diabetics have a higher risk factor profile and poor clinical outcome.

Early diagnosis and appropriate management will reduce the risk of complication after the onset of disease.

\section{Limitation of the study}

- Study Sample: Due to the time constrains only 75 Diabetic and 125 Non diabetics were studied.

- Management of CAD has not been studied, which requires further research.

\section{References}

1. Fein F, Scheuer J. Heart disease in diabetes. In: Rifkin H Jr, ed. Diabetes Mellitus: Theory and Practice. New York, NY: Elsevier Science Publishing Co Inc; 1990:812-823

2. Usitupa M, Siitonen O, Aro A. Prevalence of coronary heart disease, left ventricular failure and hypertension in middle-aged, newly diagnosed type 2 (non-insul
independent)
diabetic
subjects.

Diabetologia. 1985;28:22- 27

3. Bryfogle JW, Bradley RF. The vascular complications of diabetes mellitus. Diabetes. 1957;6:159-167

4. Anderson RS, Ellington A, Gunter LM. The incidence of arteriosclerotic heart disease in negro diabetic patients. Diabetes. 1961;10:114-118

5. Epstein FH, Ostrander LD Jr, Johnson BC, Payne MW, Hayner NS, Keller JB, Francis T. Epidemiological studies of cardiovascular disease in a total community: Tecumseh, Michigan. Ann Intern Med. 1965;62:1170- 1187

6. Kannel WB, Dawber TR, Kagan A, Revotskie N, Stokes J. Factors of risk in the development of coronary heart disease: sixyear follow-up experience:

7. Haffner SM, Lehto S, Ronnemaa T, et al. Mortality from coronary heart disease in subject with type 2 diabetes and in nondiabetic subjects with and without prior myocardial infarction. NEng $J$ of med.1998;339:229- 234

8. Laing SP, Swerdlow AJ, Slater SD, etal. Mortality from heart disease in a cohort of 23000 patients with insulin treated diabetes.Diabetologia.2003;46:760- 765

9. Geiss LS, Herman WH, Smith PJ. Mortality in noninsulin dependent diabetes. In: Diabetes in America.2nd ed. National Diabetes Data Group, National institutes of Health, National Institute of Diabetes and Digestive and Kidney Disease, NIH publication No.95-1468,1995; 233-257

10. Hamby R, Sherman L, Mehta J, Aintablian A. Reappraisal of the role of the diabetic state in coronary artery disease. Chest. 1976;70:251-257

11. Waller B, Palumbo P, Roberts W. Status of the coronary arteries at necropsy in diabetes mellitus with onset after age 30 years. Am J Med. 1980;69:498-506 
12. Fein F. Heart disease in diabetes. Cardiovasc Rev Rep. 1982;3:877-893

13. Rosove M, Harrison F, Harwig M. Plasma Bthromboglobulin, platelet factor 4, fibrinopeptide $\mathrm{A}$, and other hemostatic functions during improved short-term glycemic control in diabetes mellitus. Diabetes Care. 1984;7:174-179

14. MacRury S, Lowe G. Blood rheology in diabetes mellitus. Diabet Med. 1990;7:285291

15. Breddin H, Krzywanek H, Althoff $P$, Schoffling K, Ubeila K. PARD: Platelet aggregation as a risk factor in diabetes: Results of a prospective study.HormMetab Res. 1985;15:63-68

16. https:/www.nhlbi.nih.gov/ files/docs/ guidelines/ express.pdf

17. http://www.ndei.org/ uploaded Files/ Common/ NDEI/ Treatment_Guidelines/ ADA\%202015\%20Summary\%20PDF.pdf

18. Nathan DM, Meigs J, Singer DE. The Epidemiology of cardiovascular disease in Type 2 diabetes mellitus: How sweat it is/or is it. The Lancet 1997;350(s1):s14-8.

19. Mak KH, Moliterno DJ, Granger CB, Miller DP, White HD, Wilcox RG, et al. Influence of Diabetes Mellitus on Clinical Outcome in the thrombolytic era of Acute Myocardial Infarction-GUSTO-1 Investigations J. Am. Coll. Cardiol. 1997;30:171-9

20. Moreno Pedro R, Murcia Alvaro M, Palacios Igor F, Leon Miltiadis N, Bernardi Victor H, FusterValentín, Fallon John T. Coronary Composition and Macrophage Infiltration in Atherectomy Specimens From Patients With Diabetes Mellitus. J of the American Heart Association October 2000; 102 (18): 2180-2184

21. Jose A. Silva, Alvaro Escobar,Tyrone J. Collins, Stephen R. Ramee, Christopher J. White. Unstable Angina, A Comparison of Angioscopic Findings Between diabetic and nondiabetic Patients. j of American Heart Association. 1995;92:1731-1736
22. Henry P, Makowski S, Richard P, Beverelli F, Casanova S, Louali A, et al. Increased incidence of moderate stenosis among patients with diabetes: Substrate for myocardial infarction? Am Heart J 1997;134:1037-43.

23. Sousa JM, Herrman JL, Teodoro M, Diogo S, Terceiro BB, Paola AA, et al. Comparison of coronary angiography findings in diabetic and non-diabetic women with non-ST-segment-elevation acute coronary syndrome. Arq Bras Cardiol 2006;86:150-5.

24. Mossavi M, Nematipour E, Mehrpooya M. Comparison of extent of coronary artery disease in angiography of diabetics and nondiabetics. Iran Heart J 2006;7:37-42.

25. Uddin SN, Malik F, Bari MA, Siddiqui NI, Khan GK, Rahman S, et al. Angiographic severity and extent of coronary artery disease in patients with type 2 diabetes mellitus. Mymensingh Med J 2005;14:32-7.

26. Nicholls SJ, Tuzcu EM, Kalidindi S, Wolski $\mathrm{K}$, Moon KW, Sipahi I, et al. Effect of diabetes on progression of coronary atherosclerosis and arterial remodeling: A pooled analysis of 5 intravascular ultrasound trials. J Am Coll Cardiol 2008;52:255-62.

27. Rana JS, Dunning A, Achenbach S, AlMallah M, Budoff MJ, Cademartiri F, et al. Difference in prevalence, extent, severity and prognosis of coronary artery disease among patients with and without diabetes undergoing coronary computed tomography angiography. Diabetes Care 2012;35:178794.

28. Fox CS, Sullivan L, D'Agostino RB Sr, Wilson PW; Framingham Heart Study. The significant effect of diabetes duration on coronary heart disease mortality: The Framingham Heart Study. Diabetes Care 2004;27:704-8. 